\title{
Chinese Cervicocephalic artery dissection study (CCADS): rationale and protocol for a multicenter prospective cohort study
}

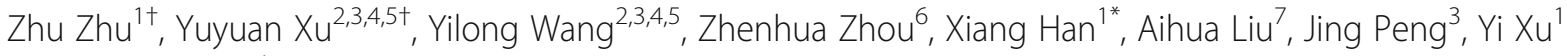
and Luyao Wang ${ }^{4}$

\begin{abstract}
Background: Cervicocephalic artery dissection (CAD) is an important etiology of stroke in the youth. Findings from recent studies suggest it a "group of disease entities" with different underlying etiologies, presentations and prognosis, necessitating an integral study including various types of CAD to get a better understanding of this disease. In addition, Chinese patients with CAD are likely to carry different features from their western counterparts, which remains uncertain yet. Chinese Cervicocephalic Artery Dissection Study (CCADS) therefore aims at exploring the epidemiology, risk factors, clinical/radiological features, diagnosis and prognosis of CAD in Chinese patients.

Methods/design: CCADS is a multicenter prospective cohort study enrolling patients age $\geq 18$ years with recent ( $<14$ days after onset) CAD. Baseline clinical data, laboratory tests and imaging studies are performed within 3 days after admission, and follow-ups will be conducted through face-to-face interviews at discharge, 3 months, 6 months and 12 months after admission, when the modified Rankin Scale (mRS), cerebrovascular events, medication compliance, CAD evolution and so on are evaluated. Additional blood samples will also be collected at baseline, 3 and 12 months follow-up. The primary outcome is radiographic evolution of CAD; secondary outcomes include cerebrovascular events, major bleeding complications, all-cause mortality and functional independence.

Discussion: Through the integration of information on epidemiology, risk factors, clinical/radiological features and prognosis of various types of CAD in Chinese population, combined with the application of advanced imaging techniques, collection of potential blood biomarkers, and assessment of novel treatment strategies. CCADS will provide thorough information on CAD - the major cause of stroke in the youth, and play a role in prevention and treatment determination in the future.
\end{abstract}

Keywords: Cervicocephalic artery dissection, Cohort, Risk factors, Magnetic resonance imaging, Biomarker, Prognosis

\section{Background}

Artery dissection develops when blood enters the wall of a vessel and separates the layers, which can be caused by a tear in the intimal layer or rupture of the vaso vasorum in the media [1]. Cervicocephalic artery dissection $(\mathrm{CAD})$ is an important etiology of stroke in the youth, accounting for $8 \%-25 \%$ of stroke events among patients aged 18 to 49 years [2, 3]. Thanks to the

\footnotetext{
* Correspondence: hansletter@fudan.edu.cn

${ }^{\dagger}$ Equal contributors

'Department of Neurology, State Key Laboratory of Medical Neurobiology,

Huashan Hospital, Fudan University, Shanghai, China

Full list of author information is available at the end of the article
}

development of advanced imaging techniques, CAD has been increasingly recognized in the past few years, some aspects of this disease, however, remain mysterious to researchers.

Although CAD used to be considered a "single disease", findings from recent studies suggest it a "group of disease entities" with various features based on different sites (intracranial vs extracranial, anterior vs posterior circulation), morphologies (intimal flap, double lumen or intramural hematoma), and presentations (isolated headache, cranial nerve palsies, ischemic stroke or subarachnoid hemorrhage) [4-6]. CADISP (Cervical 
Artery Dissection and Ischemic Stroke Patients) is the largest cohort study worldwide examining different profiles according to the dissection site in ischemic stroke patients with cervical artery dissection [7], while knowledge on either milder (asymptomatic) or more severe (intracranial artery dissection presenting as subarachnoid hemorrhage) type of CAD is lacking. Thus, to establish a cohort including various kinds of CAD may be helpful for identifying both commonness and individuality of this disease.

In addition, despite the overall recurrence rate of stroke induced by artery dissection being low [8, 9], the evolution of artery dissection itself remains uncertain, especially according to different treatments, underlying etiologies and clinical or morphological features. By assuming that the existence of CAD may worsen the vascular lesions in the long run particularly when atherosclerosis develops with aging, not only the clinical outcomes but also the radiographic evolution of CAD needs to be paid close attention to.

Finally, Chinese patients with cerebrovascular diseases have been reported to carry different profiles compared with their western counterparts [10]. The characteristics of CAD associated stroke in Chinese population, however, remain uncertain. Data from previous single-center research with relatively small sample size indicated higher proportion of intracranial artery dissection and posterior circulation involvement in Chinese CAD patients $[11,12]$. These features, on one hand, make the diagnosis of CAD more challenging because of small lumen and tortuous course of involved vessels; on the other hand, carry high risk of hemorrhagic transformation when antithrombotic treatments are administered. At present, the diagnosis and management of CAD in China are largely based on specialist consensus due to lack of high-class evidence [13], thus necessitating the establishment of a Chinese cohort with large sample size for better clinical practice in the future.

Therefore, we describe here the protocol of CCADS, a multicenter prospective cohort study to explore the epidemiology, risk factors, clinical/radiological features, diagnosis and prognosis of CAD in Chinese patients incorporating the application of advanced imaging techniques, collection of potential blood biomarkers, and assessment of novel treatment strategies.

\section{Methods/design}

\section{Study design}

CCADS is a national, multicenter, consecutive, prospective, cohort study enrolling patients with cervical or intracranial artery dissection within 14 days after symptom onset. Patient recruitment will take place at 56 centers in China. Approval of the Ethics Committee at each center has been obtained and all participants or their next of kin provide written consent at the time of enrollment in the cohort.
The total duration of the study will be approximately 4 years, from September 2017 (first in) until August 2021 (last out).

\section{Study objectives}

The primary objective of this study is to get a better knowledge on epidemiology, risk factors, clinical presentations and prognosis of CAD in Chinese population by setting up a large cohort; to assess the effect of different treatment strategies on clinical and radiographic evolution of CAD. Secondary objectives include to: 1) find out the association between possible etiologies (e.g. hereditary diseases) or risk factors (e.g. infection) and characteristics of CAD (e.g. multiple dissections, dissecting aneurysm, etc.); 2) determine the relationship between baseline features, functional outcomes and radiographic evolution of CAD; 3 ) establish standardized process for early and accurate diagnosis of CAD by analyzing relatively specific clinical and radiological features; 4) investigate individualized therapies, laying a foundation for further clinical trials.

\section{Study population}

All consecutive patients referred to the department of Neurology of 56 tertiary teaching hospitals and regional hospitals (see Additional file 1) in China with clinical or imaging work-ups suggesting CAD will be screened for eligibility. Patients fulfilling the inclusion and not the exclusion criteria are asked to participate.

Inclusion criteria:

1. Age 18 years or older.

2. Within 14 days after the first symptom onset for symptomatic patients; or asymptomatic patients.

3. Typical radiological characteristics in at least one confirmatory angiographic examination including magnetic resonance angiography (MRA), computerized tomographic angiography (CTA), or digital subtraction angiography(DSA): intimal flap, double lumen, dissecting aneurysm or luminal dilation plus stenosis. For an artery exhibiting nonspecific stenosis or occlusion, CAD will be diagnosed if cross-sectional MRI demonstrated intramural hematoma and intraplaque hemorrhage is excluded $[5,14]$.

\section{Exclusion criteria:}

1. Pregnant women.

2. Patients refusing to participate the study.

\section{Procedures}

Eligible patients will be recruited at the time of confirmed diagnosis of CAD. We intend to include 1300 
patients, which is expected to complete over a 3-year period. Baseline assessments including clinical data, laboratory tests and imaging studies are performed within 3 days after admission, and a blood sample will be drawn from each patient during the time in hospital. Follow-ups will be conducted through faceto-face interviews at discharge, 3 months, 6 months and 12 months after admission, when the modified Rankin Scale (mRS), cerebrovascular events, medication compliance, CAD evolution and so on are going to be evaluated by trained neurologists. A flowchart of the study is shown in Fig. 1.

\section{Study measurements}

\section{Clinical data}

Data of patients will be collected using an electronic Case Report Form (e-CRF) at baseline and during the follow-up period. Clinical items recorded include demographics, medical history, clinical presentations, physical examination findings and treatments in hospital. The detailed information is shown in Table 1.

\section{Laboratory tests and blood sample collection}

Clinical biochemistry tests including total blood cell count, renal and liver functions, glucose and lipid levels, folic acid, Vitamin B12, homocysteine concentrations and autoimmune biomarkers will be measured using standard lab procedures within 3 days after admission and recorded at discharge of the patients.

Three additional blood samples (containing plasma, serum and white cells, respectively) will also be taken from the peripheral vein of each participant at baseline, 3 and 12 months follow-up. All the samples will be collected into tubes and stored at $-20{ }^{\circ} \mathrm{C}$ until use for future pooled analysis.

\section{Imaging protocols}

MRI

The MRI studies are performed on a 3 Tesla MRscanner (DISCOVERY, MR750, GE Medical systems, Milwaukee or MAGNETOM Verio, SIEMENS Medical Systems, Germany). The scanning protocol include T1, T2, T2 fluid attenuated inversion recovery (FLAIR) and

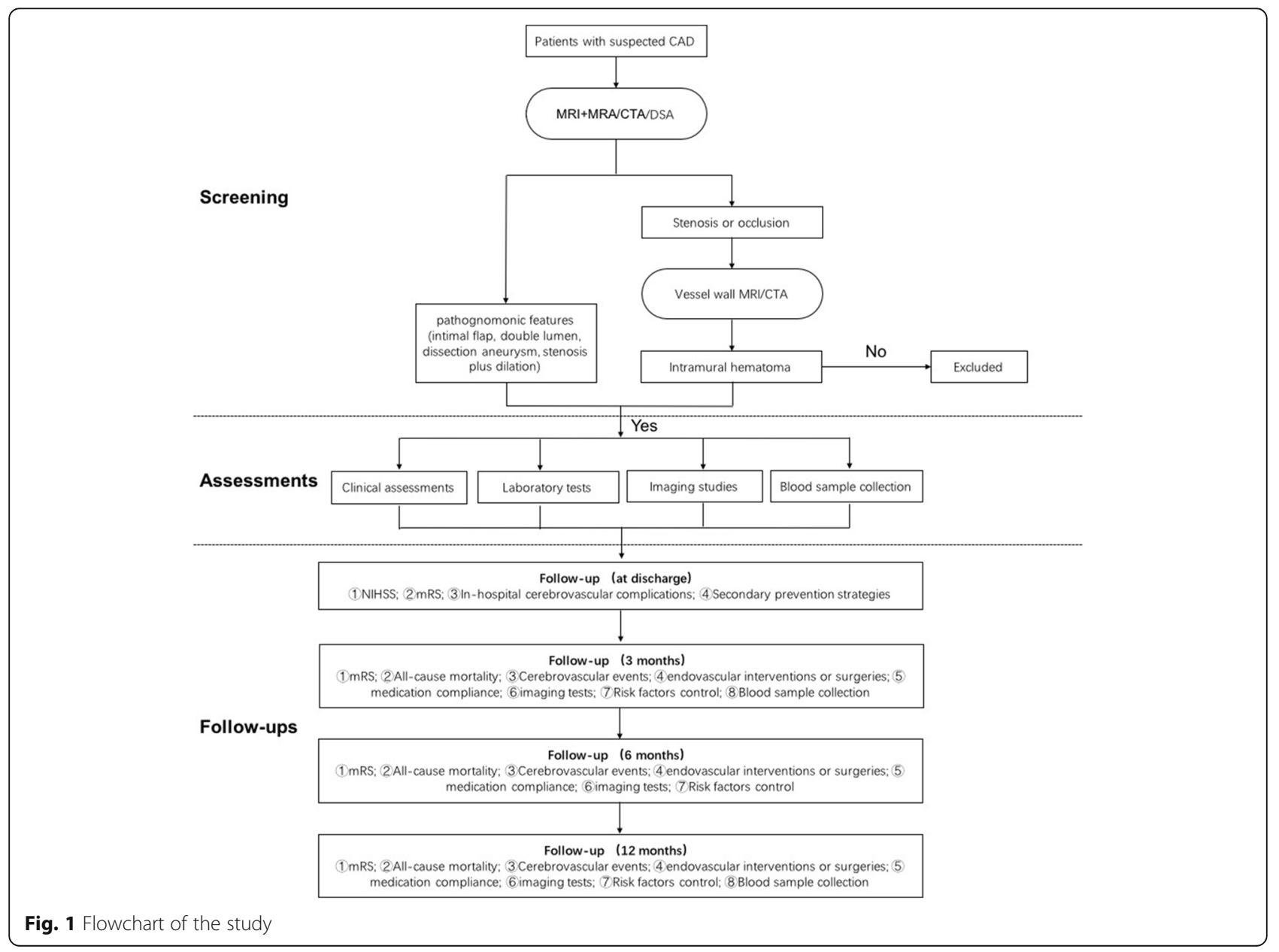


Table 1 Clinical assessments for CAD patients in CCADS

\begin{tabular}{|c|c|}
\hline Domain & Assessment \\
\hline Demographics & age, gender, educational level, contact information \\
\hline \multicolumn{2}{|l|}{ Medical history } \\
\hline $\begin{array}{l}\text { vascular risk } \\
\text { factors }\end{array}$ & American Heart Association guideline [21] \\
\hline migraine & $\begin{array}{l}\text { classification and diagnostic criteria for headache } \\
\text { disorders, cranial neuralgias and facial pain [22] }\end{array}$ \\
\hline I CTD* & $\begin{array}{l}\text { Ehlers-Danlos syndrome, Marfan Syndrome, } \\
\text { Loeys-Dietz syndrome, etc. [23] }\end{array}$ \\
\hline $\begin{array}{l}\text { autoimmune } \\
\text { diseases }\end{array}$ & SLE, Becet's disease, Sjogren's syndrome, etc. \\
\hline mild trauma & $\begin{array}{l}\text { neck manipulation or overextension } \\
\text { (within } 2 \text { weeks prior to symptom onset) [24] }\end{array}$ \\
\hline infection & within 2 weeks prior to symptom onset \\
\hline \multicolumn{2}{|l|}{ Personal history } \\
\hline smoking & a structured questionnaire embedded in CRF \\
\hline alcohol use & a structured questionnaire embedded in CRF \\
\hline risk factor control & hypertension, migraine, etc. \\
\hline \multicolumn{2}{|l|}{ Clinical presentations } \\
\hline stroke & $\begin{array}{l}\text { clinical stroke syndrome (sudden neurological } \\
\text { dysfunction lasting }>24 \text { h, with no apparent } \\
\text { cause other than that of vascular origin) }\end{array}$ \\
\hline TIA & $\begin{array}{l}\text { rapidly evolving focal neurological deficit, } \\
\text { without positive phenomena such as twitches, } \\
\text { jerks or myoclonus, with no other than vascular } \\
\text { cause lasting less than } 24 \mathrm{~h}\end{array}$ \\
\hline cranial nerve palsy & $\begin{array}{l}\text { peripheral hypoglossal nerve or facial nerve } \\
\text { palsy }\end{array}$ \\
\hline headache & $\begin{array}{l}\text { new onset headache; severity, nature, location } \\
\text { or frequency change }\end{array}$ \\
\hline Horner syndrome & Miosis, partial ptosis, loss of hemifacial sweating \\
\hline stroke severity & NIHSS [25] \\
\hline stoke risk after TIA & ABCD2 scores [26] \\
\hline $\begin{array}{l}\text { Functional } \\
\text { independence }\end{array}$ & mRS [27] \\
\hline \multicolumn{2}{|l|}{ Treatment } \\
\hline $\begin{array}{l}\text { antithrombotic } \\
\text { drugs }\end{array}$ & aspirin, clopidogrel, NOAC \\
\hline $\begin{array}{l}\text { reperfusion } \\
\text { therapy }\end{array}$ & $\begin{array}{l}\text { intravenous thrombolysis, intra-arterial } \\
\text { thrombolysis, endovascular intervention }\end{array}$ \\
\hline other medicines & statin, antihypertensive therapy, antidiabetics \\
\hline $\begin{array}{l}\text { medication } \\
\text { compliance }\end{array}$ & a structured questionnaire \\
\hline time & $\begin{array}{l}\text { time from symptom onset to treatment, and } \\
\text { treatment duration }\end{array}$ \\
\hline
\end{tabular}

*ICTD Inherited connective tissue disorders, mRS Modified Rankin Scale, NIHSS National Institute of Health Stroke Scale, TIA Transient ischemic attack, NOAC Novel oral anticoagulant, SLE Systemic lupus erythematosus

diffusion weighted imaging (DWI) sequences of the brain. Vascular MRI scanning is performed using standardized protocol consisting of magnetic resonance angiography (MRA) and high-resolution vessel wall imaging, which have been recommended for detection of CAD $[6,15,16]$. The corresponding sequences are described in Table 2.

DSA DSA is performed via transfemoral approach with intra-arterial injection of contrast medium, and all images are acquired on an Infinix (Toshiba Medical Systems, Tokyo, Japan) imaging system. Selective catheterization of the internal carotid arteries and the vertebral arteries are going to be done. Standard anteroposterior and lateral projection images, as well as magnified oblique projections, are obtained. For suspected artery dissection, rotational 3D angiography is also done to better delineate the details of the artery.

CTA CTA is performed using 256-row CT scanners (Brilliance iCT, Royal Philips Electronics, NEDERLAND B.V.) with the scanning range from aortic arch to vertex (slice thickness: $0.9 \mathrm{~mm}$; reconstruction interval: $0.7 \mathrm{~mm}$; voltage: $100 \mathrm{Kv}$; current: $125 \mathrm{mAs}$; FOV: $220 \mathrm{~mm}$ ). Ioxehol is injected from antecubital vein at a rate of $5 \mathrm{ml} / \mathrm{s}$ for a total of $50 \mathrm{ml}$ in volume. Images will be transferred to postprocessing workstation (Extended Brilliance Workspace, ver. 4.5, Philips) for further analysis and reconstruction. Maximum intensity projection (MIP), multiplaner reformations (MPR) and volume rendered (VR) techniques are applied to reconstruct raw image data. Both raw data and reconstructed models are used for evaluation.

\section{Image analysis and classification}

Morphologies of CAD are grouped into intimal flap (a layer crossing the arterial lumen), double lumen (true plus false lumens), dissection aneurysm (luminal dilation), pearl-and-string sign (aneurysmal dilatation alternating with stenosis) and tapered steno-occlusion plus evidence of intramural hematoma (eccentric, intermediate-to-high signal intensity of the arterial wall according to hemorrhagic age). The proximal part (entry point) of a dissection lesion is defined as the site of artery dissection. As such, a dissection located completely within the cranium is classified as intracranial dissection, and a dissection starting extracranially as extracranial dissection. Multiple dissections will be recorded when more than two different arteries are involved; single dissection is recognized when the lesion localized in one vessel regardless of the length.

Two experienced neurologists will evaluate baseline and follow-up images independently at each center, and images will be reviewed centrally by 3 principle investigators (YL Wang, X Han, ZH Zhou) of the study in case of disagreement. 
Table 2 MRI parameters of vascular imaging

\begin{tabular}{|c|c|c|c|c|c|c|c|}
\hline Scanner & Sequences & TR/TE (ms) & $\mathrm{FOV}(\mathrm{mm})$ & Matrix & Number of slices & Slice thickness(mm) & time \\
\hline \multirow[t]{3}{*}{ GE } & 3D TOF & $25 / 3.4$ & $220 \times 200$ & $320 \times 192$ & 96 & 1.4 & $3: 32$ \\
\hline & 2D FSE & $2500 / 85$ & $150 \times 150$ & $384 \times 256$ & 12 & 2 & $2: 45$ \\
\hline & 3D CUBE & $350 / 15$ & $200 \times 180$ & $256 \times 192$ & 32 & 1 & $2: 46$ \\
\hline \multirow[t]{3}{*}{ SIEMENS } & 3D TOF & $21 / 3.6$ & $200 \times 180$ & $256 \times 224$ & 120 & 0.9 & $2: 37$ \\
\hline & 2D FSE & $1500 / 26$ & $150 \times 150$ & $256 \times 256$ & 20 & 2 & $3: 48$ \\
\hline & 3D SPACE & $1500 / 252$ & $200 \times 180$ & $256 \times 224$ & 56 & 0.8 & $4: 14$ \\
\hline
\end{tabular}

TR Repetition time, TE Echo time, FOV Field of view, Matrix Frequency $\mathrm{x}$ phase, TOF Time of flight, FSE Fast spin echo

\section{Outcome measures}

The primary outcome of this study is the radiographic evolution of CAD, which is classified as complete recovery, partial recovery, unchanged, or progression by comparing the radiological features of dissected arteries in follow-up period with those at baseline. Secondary outcomes include cerebrovascular events (ischemic or hemorrhagic stroke, TIA), major bleeding complications according to PLATO (Platelet Inhibition and Patient Outcomes) definition [17], all-cause mortality and functional independence assessed by mRS.

\section{Sample size and analysis}

This is a cohort study without specific hypothesis concerning the primary outcome. So sample size is calculated based on the method recommended for registry study (10 times number of variable) [18], with the estimated number of variables as 110 . The sample size is therefore of at least 1100, which is increased to 1300 considering possible losses.

Statistical analysis will be performed using SAS statistical package. Statistical significance for previously mentioned outcomes will be assessed using $\chi^{2}$ or Fisher's exact test for categorical variables such as vascular risk factors, genders, treatment strategies, and t-test, ANOVA, Mann-Whitney U or Kruskal-Wallis test for continuous variables like age, NIHSS, etc. Logistic regression models will be applied to determine potential risk factors of CAD; Cox proportional hazards multivariate analysis will then be performed to identify clinical and radiological predictors of outcomes in patients with CAD, adjusted by variables with $P<0.1$ on univariate analysis. The correlation between continuous variables will be tested with Spearman's or Pearson's coefficients, where appropriate. Two-tailed $P<0.05$ is considered significant.

\section{Discussion}

CCADS is a nationwide prospective study focusing on CAD - a probably underrecognized while definitely innegligible culprit of young stroke. The differences of this study from previous ones lie in:
First, both cervical and intracranial artery dissection will be included, with the latter type still far from being fully understood at present because of the very low prevalence worldwide $[19,20]$. The findings of CCADS, therefore, may help get a better understanding of this disease. Similarly, the coverage of various types of artery dissection (anterior and posterior circulation, symptomatic and asymptomatic, stenosis/occlusion and dissecting aneurysm lesions) in combination with large sample size can provide comprehensive as well as characteristic information on CAD, hence laying foundation for future clinical trials on individualized management.

Second, in addition to cerebrovascular events and functional independence, the radiographic evolution of CAD will be also specially followed up, and the predictors associated with anatomic recovery or progression are going to be assessed in this study.

Third, additional blood samples will be collected at acute and chronic stages of CAD, the analysis of which may help find out potential biomarkers for early diagnosis or for mechanism investigations.

Finally, the large Chinese cohort carries both specific and common features of CAD, providing more thorough information on this important etiology leading to stroke in the youth, which will play a role in prevention and treatment determination in the future.

\section{Additional file}

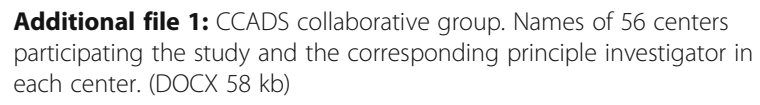

Additional file 1: CCADS collaborative group. Names of 56 centers participating the study and the corresponding principle investigator in each center. (DOCX $58 \mathrm{~kb}$ )

\footnotetext{
Abbreviations

3D: Three dimensional; CAD: Cervicocephalic artery dissection; CADISP: Cervical artery dissection and ischemic stroke patients; CTA: Computed tomography angiography; DSA: Digital subtraction angiography; DWl: Diffusion weighted imaging; e-CRF: Electronic case report form; FLAIR: Fluid attenuated inversion recovery; FOV: Field of view; FSE: Fast spin echo; ICTD: Inherited connective tissue disorders; MIP: Maximum intensity projection; MPR: Multiplaner reformations; MRA: Magnetic resonance angiography; MRI: Magnetic resonance imaging; mRS: Modified Rankin scale; NIHSS: National institute of health stroke scale; NOAC: Novel oral anticoagulant; PLATO: Platelet inhibition and patient outcomes; SAH: Subarachnoid hemorrhage; SLE: Systemic lupus erythematosus; TE: Echo
} 
time; TIA: Transient ischemic attack; TOF: Time of flight; TR: Repetition time; VR: Volume rendered

\section{Acknowledgements}

We would like to thank all researchers, institutions and patients for their collaboration and contribution to this study. We thank the Chinese Stroke Association for their invaluable support of this study.

\section{Funding}

Not applicable.

\section{Availability of data and materials}

Data sharing is not applicable to this article as no datasets were generated or analyzed for this manuscript. The CCADS data will be available from the corresponding author on reasonable request after the study completed.

\section{Authors' contributions}

$Z Z$ and $Y X$ collected the data, wrote the manuscript and contributed to the design of the study. $\mathrm{YW}, \mathrm{XH}$ and $\mathrm{ZZ}$ conceived of the study, conducted the data analysis and assisted in drafting the manuscript. AL contributed to the study design and critical review of manuscript. JP, YX and LW: collected the data, critically read the manuscript. All authors read and approved the final manuscript.

\section{Ethics approval and consent to participate}

Approval of the Ethics Committee at each center has been obtained and all participants or their next of kin provide written consent at the time of enrollment in the cohort.

\section{Consent for publication}

$$
\text { Not applicable. }
$$

\section{Competing interests}

The authors declare that they have no competing interests.

\section{Publisher's Note}

Springer Nature remains neutral with regard to jurisdictional claims in published maps and institutional affiliations.

\section{Author details}

'Department of Neurology, State Key Laboratory of Medical Neurobiology, Huashan Hospital, Fudan University, Shanghai, China. ${ }^{2}$ Department of Neurology, Beijing Tiantan Hospital, Capital Medical University, Beijing, China. ${ }^{3}$ China National Clinical Research Center for Neurological Diseases, Beijing, China. ${ }^{4}$ Center of Stroke, Beijing Institute for Brain Disorders, Beijing, China. ${ }^{5}$ Beijing Key Laboratory of Translational Medicine for Cerebrovascular Disease, Beijing, China. ${ }^{6}$ Department of Neurology, Southwest Hospital, Third Military Medical University, Chongqing, China. 'Department of Interventional Neuroradiology, Beijing Tiantan Hospital, Capital Medical University, China; Beijing Neurosurgical Institute, Capital Medical University, Beijing, China.

\section{Received: 21 August 2017 Accepted: 3 January 2018}

\section{Published online: 11 January 2018}

\section{References}

1. Shah Q, Messe SR. Cervicocranial arterial dissection. Curr Treat Options Neurol. 2007;9(1):55-62.

2. Menon RK, Norris JW. Cervical arterial dissection: current concepts. Ann N Y Acad Sci. 2008;1142:200-17.

3. Debette $\mathrm{S}$, Leys D. Cervical-artery dissections: predisposing factors, diagnosis, and outcome. The Lancet Neurology. 2009;8(7):668-78.

4. Bejot Y, Daubail B, Debette S, Durier J, Giroud M. Incidence and outcome of cerebrovascular events related to cervical artery dissection: the Dijon stroke registry. Int J Stroke. 2014;9(7):879-82.

5. von Babo M, De Marchis GM, Sarikaya H, Stapf C, Buffon F, Fischer U, Heldner MR, Gralla J, Jung S, Simonetti BG, et al. Differences and similarities between spontaneous dissections of the internal carotid artery and the vertebral artery. Stroke. 2013;44(6):1537-42.

6. Debette $\mathrm{S}$, Compter A, Labeyrie MA, Uyttenboogaart M, Metso TM, Majersik $J$ J, Goeggel-Simonetti B, Engelter ST, Pezzini A, Bijlenga P, et al.
Epidemiology, pathophysiology, diagnosis, and management of intracranial artery dissection. The Lancet Neurology. 2015;14(6):640-54.

7. Debette S, Grond-Ginsbach C, Bodenant M, Kloss M, Engelter S, Metso T, Pezzini A, Brandt T, Caso V, Touze E, et al. Differential features of carotid and vertebral artery dissections: the CADISP study. Neurology. 2011;77(12):1174-81.

8. Ct i, Markus HS, Hayter E, Levi C, Feldman A, Venables G, Norris J. Antiplatelet treatment compared with anticoagulation treatment for cervical artery dissection (CADISS): a randomised trial. The Lancet Neurology. 2015;14(4):361-7.

9. Engelter ST, Brandt T, Debette S, Caso V, Lichy C, Pezzini A, Abboud S, Bersano A, Dittrich R, Grond-Ginsbach C, et al. Antiplatelets versus anticoagulation in cervical artery dissection. Stroke. 2007:38(9):2605-11.

10. Tsai CF, Thomas B, Sudlow CL. Epidemiology of stroke and its subtypes in Chinese vs white populations: a systematic review. Neurology. 2013; 81(3):264-72

11. Zhao KJ, Zhao R, Huang QH, XU Y, Hong B, Fang YB, Li Q, Yang PF, Liu JM, Zhao WY. The interaction between stent(s) implantation, PICA involvement, and immediate occlusion degree affect symptomatic intracranial spontaneous vertebral artery dissection aneurysm (sis-VADA) recurrence after reconstructive treatment with stent(s)-assisted coiling. Eur Radiol. 2014; 24(9):2088-96

12. Li H, Zhang X, Li XF, He XY, Zhu GH, Fang QR, Wang ZQ, Duan CZ. Predictors of favorable outcome of intracranial basilar dissecting aneurysm. Journal of stroke and cerebrovascular diseases : the official journal of National Stroke Association. 2015;24(8):1951-6.

13. Wang Y-M, Zhang Y-S, Sui B-B, Yang X-J, Gao P-Y. Chinese specialist consensus on imaging diagnosis of intracranial arterial dissection. Chinese Neurosurgical Journal. 2017;3(1)

14. Zhang FL, Liu Y, Xing YQ, Yang Y. Diagnosis of cervical artery dissection using 3-T magnetic resonance imaging. JAMA neurology. 2015;72(5):600-1.

15. Swartz RH, Bhuta SS, Farb RI, Agid R, Willinsky RA, Terbrugge KG, Butany J, Wasserman BA, Johnstone DM, Silver FL, et al. Intracranial arterial wall imaging using high-resolution 3-tesla contrast-enhanced MRI. Neurology. 2009;72(7):627-34

16. Edjlali M, Roca P, Rabrait C, Naggara O, Oppenheim C. 3D fast spin-echo T1 black-blood imaging for the diagnosis of cervical artery dissection. AJNR Am J Neuroradiol. 2013;34(9):E103-6.

17. Wallentin $L$, Becker RC, Budaj A, Cannon CP, Emanuelsson H, Held C, Horrow J, Husted S, James S, Katus H, et al. Ticagrelor versus clopidogrel in patients with acute coronary syndromes. N Engl J Med. 2009;361(11):1045-57.

18. Noordzij M, Dekker FW, Zoccali C, Jager KJ. Sample size calculations. Nephron Clin Pract. 2011;118(4):C319-23.

19. Arnold M, Bousser MG, Fahrni G, Fischer U, Georgiadis D, Gandjour J, Benninger D, Sturzenegger M, Mattle HP, Baumgartner RW. Vertebral artery dissection: presenting findings and predictors of outcome. Stroke. 2006; 37(10):2499-503.

20. Arauz A, Ruiz A, Pacheco G, Rojas P, Rodriguez-Armida M, Cantu C, MurilloBonilla L, Ruiz-Sandoval JL, Barinagarrementeria F. Aspirin versus anticoagulation in intra- and extracranial vertebral artery dissection. Eur J Neurol. 2013;20(1):167-72.

21. Goldstein LB, Adams R, Alberts MJ, Appel $L$, Brass LM, Bushnell CD, Culebras A, Degraba TJ, Gorelick PB, Guyton JR, et al. Primary prevention of ischemic stroke: a guideline from the American Heart Association/American Stroke Association stroke council: cosponsored by the atherosclerotic peripheral vascular disease interdisciplinary working group; cardiovascular nursing council; clinical cardiology council; nutrition, physical activity, and metabolism council; and the quality of care and outcomes research interdisciplinary working group: the American Academy of Neurology affirms the value of this guideline. Stroke. 2006;37(6):1583-633.

22. Classification and diagnostic criteria for headache disorders. Cranial neuralgias and facial pain. Headache classification Committee of the International Headache Society. Cephalalgia : an international journal of headache. 1988;8(Suppl 7):1-96.

23. Debette $\mathrm{S}$, Germain DP. Neurologic manifestations of inherited disorders of connective tissue. Handb Clin Neurol. 2014;119:565-76.

24. Engelter ST, Grond-Ginsbach C, Metso TM, Metso AJ, Kloss M, Debette S, Leys D, Grau A, Dallongeville J, Bodenant M, et al. Cervical artery dissection: trauma and other potential mechanical trigger events. Neurology. 2013; 80(21):1950-7. 
25. Brott T, Adams HP Jr, Olinger CP, Marler JR, Barsan WG, Biller J, Spilker J, Holleran R, Eberle R, Hertzberg V, et al. Measurements of acute cerebral infarction: a clinical examination scale. Stroke. 1989;20(7):864-70.

26. Rothwell PM, Giles MF, Flossmann E, Lovelock CE, Redgrave JN, Warlow CP, Mehta Z. A simple score (ABCD) to identify individuals at high early risk of stroke after transient ischaemic attack. Lancet. 2005:366(9479):29-36.

27. Burn JP. Reliability of the modified Rankin scale. Stroke. 1992;23(3):438.

Submit your next manuscript to BioMed Central and we will help you at every step:

- We accept pre-submission inquiries

- Our selector tool helps you to find the most relevant journal

- We provide round the clock customer support

- Convenient online submission

- Thorough peer review

- Inclusion in PubMed and all major indexing services

- Maximum visibility for your research

Submit your manuscript at www.biomedcentral.com/submit
Biomed Central 